\title{
ANOTACIONES A LA TRIBU STIPEAE (GRAMINEAE) PARA ANDALUCÍA ORIENTAL (ESPAÑ)
}

\author{
Francisco María VÁZQUEZ
}

\begin{abstract}
RESUMEN. Anotaciones a la tribu Stipeae (Gramineae) para Andalucía Oriental (España). El estudio taxonómico de los materiales de la tribu Stipeae (Gramineae) procedentes de Andalucía Oriental (España), ha revelado la presencia de dos nuevos taxones, posiblemente endémicos para el área: Macrochloa tenacissima subsp. umbrosa F.M. Vázquez subsp. nov y Stipa almeriensis F.M. Vázquez $s p$. nov. Además, se incluye una nueva combinación: Macrochloa antiatlantica (Barreña, D. Rivera, Alcaraz \& Obón) F. M. Vázquez, comb. nov.
\end{abstract}

Palabras clave: Poaceae, Stipa, Macrochloa, Taxonomía, Península Ibérica.

ABSTRACT: Stipeae tribe (Gramineae) annotations for eastern Andalusia (Spain). The taxonomy study of the Stipeae tribe (Gramineae) materials from Orient Andalusia (Spain) was revealed two new taxa, possibility endemic from this area: Macrochloa tenacissima subsp. umbrosa F.M. Vázquez subsp. nov and Stipa almeriensis F.M. Vázquez sp. nov. Also, the new combination: Macrochloa antiatlantica (Barreña, D. Rivera, Alcaraz \& Obón) F. M. Vázquez, comb. nov., is proposed.

Key words: Poaceae, Stipa, Macrochloa, Taxonomy, Iberian Peninsula.

\section{INTRODUCCIÓN}

El estudio de la diversidad florística de los territorios de la Península Ibérica permite conocer con mayor exactitud el grado de riqueza de taxones con los que cuenta cada área. Las iniciativas emprendidas a nivel global con Flora Ibérica (Castroviejo et al., 1986-2005) o particulares con los estudios regionales de flora (Bolós, O de \& J. Vigo, 1984-2001; Valdés et al., 1987; Devesa, 1995; Villar et al., 1997-2001); han contribuido enormemente a dimensionar con mayor acierto la riqueza florística de la Península Ibérica.

Los estudios centrados en la Flora de Andalucía Oriental han permitido revisar una buena colección de materiales de los género Achnatherum Beauv., Celtica F.M. Vázquez $\&$ Barkworth, Macrochloa Kunth y Stipa L., dentro de la tribu Stipeae. La revisión ha revelado la presencia de 24 taxones en el territorio. Algunos endémicos como Stipa cazolensis (F.M. Vázquez \& Devesa)F.M. Vázquez, H. Scholz \& M. Sonnentag o Stipa filabrensis $\mathrm{H}$. Scholz, M. Sonnentag \& F.M.

Esta contribución es fruto de los resultados del Convenio de colaboración entre EGMASA y la Universidad de Granada para realización conjunta de Estudios sobre la Flora de Andalucia Oriental. Contrato $n^{\circ} 2001$, financiado por la Junta de Andalucía. 
Vázquez (Vázquez et al., 1999) y otros nuevos para la ciencia. El objetivo de este trabajo es poner de manifiesto, con la revisión realizada, la presencia de dos nuevos taxones de la tribu Stipeae para Andalucía Oriental.

\section{MATERIAL Y MÉTODOS}

El ámbito geográfico del estudio se ha centrado en Andalucía Oriental, concretamente en las provincias de Almería, Granada, Jaén y Málaga.

Se ha realizado una revisión taxonómica de los materiales de la tribu Stipeae conservados en los herbarios ALME, GDA, GDAC, HSS, HUAL, MA y MGC, para el territorio previamente delimitado.

El estudio taxonómico nos ha confirmado en los taxones del grupo Stipa sect. Stipa la necesidad de estudiar anatómicamente el limbo de las hojas estériles. El estudio de la histología foliar se ha realizado siguiendo la técnica previamente indicada por Devesa,1992. Una vez realizados los cortes se han visionado y pasados desde un estereomicroscopio con visión trasladada a papel.

\section{RESULTADOS}

Para hacer más fácil la exposición de los resultados encontrados se procederá a comentar de forma independiente el estudio con los taxones del género Macrochloa Kunth y Stipa section Stipa L. (Vázquez \& Barkworth, 2004).

\section{Macrochloa Kunth.}

La distribución de Macrochloa tenacissima (Loefl. ex L.) Kunth es muy amplia por todo el territorio estudiado. Se encuentra representada en casi todas las comarcas delimitadas para Andalucía Oriental. Su amplia representación no está ligada a una amplia diversidad morfológica, aunque es posible encontrar algunas variaciones locales sin entidad taxonómica como la variación en la pelosidad de la arista, la longitud de los pelos en las piezas florales, el color de las glumas, o la mayor o menor presencia de esclerénquima en las hojas (Vázquez \& Devesa, 1997). Atendiendo a las variaciones morfológicas y ecológicas encontradas para otras especies del grupo, recientemente descritas como Macrochloa antiatlantica (Barreña, D. Rivera, Alcaraz \& Obón) F. M. Vázquez, comb. nov. (Basiónimo: Stipa antiatlantica Barreña, D. Rivera, Alcaraz \& Obón, Novon 16 (1): 13 (14) (2006). Isotipo: MA!), se ha encontrado una población en el extremo occidental de la provincia de Almería que se caracteriza por la presencia de un porte muy elevado, caracteres foliares y piezas florales diferentes a los típicos del taxon Macrochloa tenacissima (Loefl. ex L.) Kunth. Apoyándonos en los caracteres que aparecen reflejados en la tabla 1, se han considerado discriminar a la población almeriense como una subespecie nueva dentro de la especie típica del género Macrochloa Kunth.

Macrochloa tenacissima subsp. umbrosa F.M. Vázquez subsp. nov.

Holotipo: ALME 17470. HS: Almería; Presa de Beninar, barranco de Río Grande, 2-5-1985, J. Guirado.

Diagnosis: Affinis subspeciei Macrochloa tenacissima subsp. tenacissima, a qua differt panicula 40-60(-70) cm non 14$35(-45) \mathrm{cm}$; glumae superior 32-40(-45) $\mathrm{mm}$ non (15-)20-30(-35) mm; glumae inferior 40-47(-52) mm non (18-)22-35(-40) mm; et columnae aristae $1 / 2$ usque $2 / 3$ villosae non plene usque $2 / 3$ villosae.

Descripción: Plantas cespitosas de 170$210 \mathrm{~cm}$; vaina de la hoja escábrida o glabra; lámina 45-90 cm, convoluta, glabra en el haz y escábrida en el envés; lígula formada por 


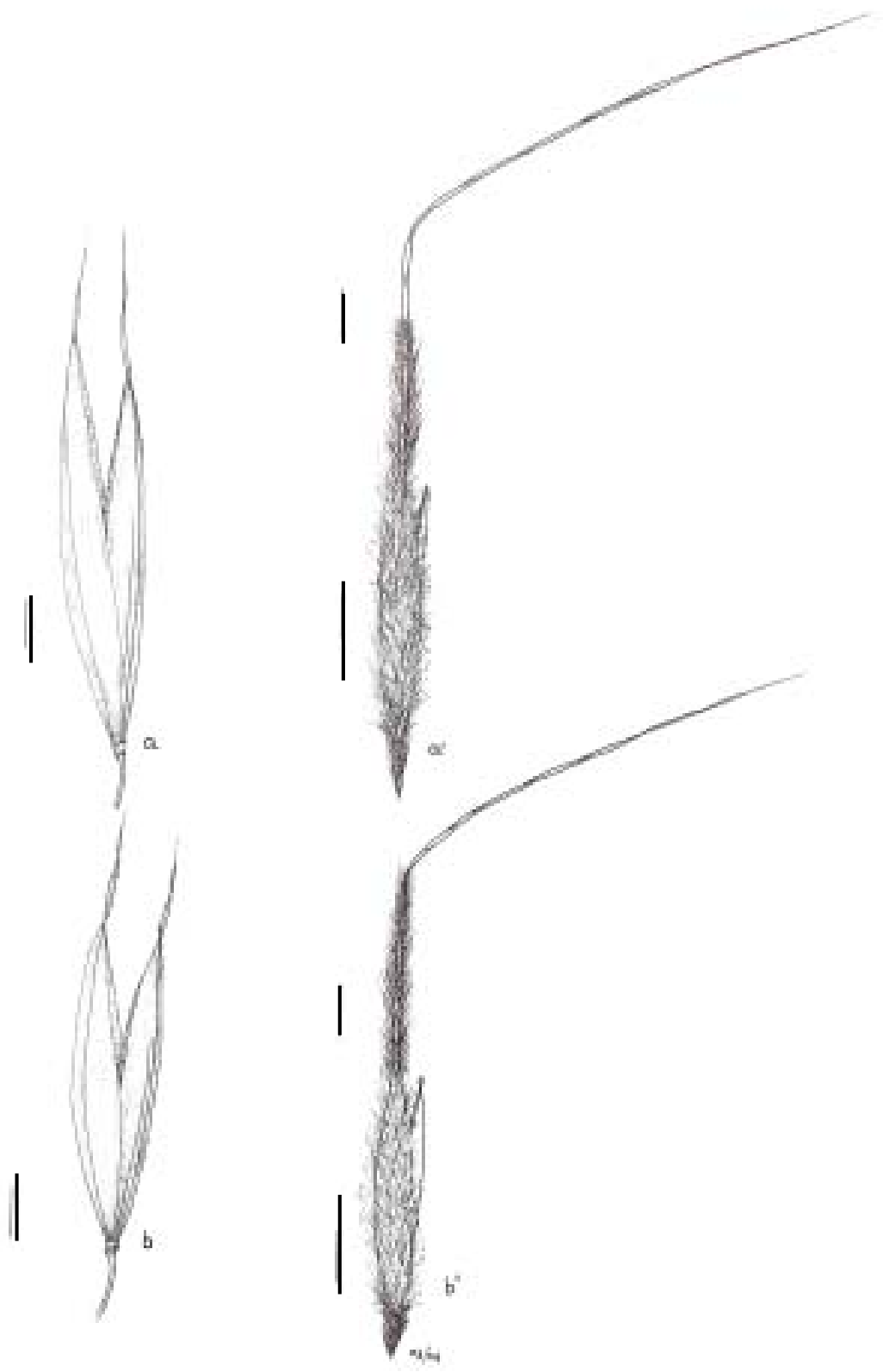

Figura 1. Glumas y antecio con arista de Macrochloa tenacissima subsp. tenacissima (Loefl. ex L.) Kunth (b y b') y Macrochloa tenacissima subsp. umbrosa F.M. Vázquez subsp. nov (a y a'). Las barras indican $4 \mathrm{~mm}$. Glumes, anthecium and awn of Macrochloa tenacissima subsp. tenacissima (Loefl. ex L.)Kunth (b and b') and Macrochloa tenacissima subsp. umbrosa F.M. Vázquez subsp. nov ( $a$ and $\left.a^{\prime}\right)$. The bars are $4 \mathrm{~mm}$. 


\begin{tabular}{lccc}
\hline \multicolumn{2}{c}{ Características } & M. tenacissima subsp. tenacissima & M. tenacissima subsp. umbrosa \\
\hline Panícula & Longitud $(\mathrm{cm})$ & $15-40(-45)$ & $40-60(-70)$ \\
Glumas & $\begin{array}{l}\text { Longitud de la } \\
\text { superior }(\mathrm{mm})\end{array}$ & $(15-) 20-30(-35)$ & $32-40(-45)$ \\
& $\begin{array}{l}\text { Longitud de la } \\
\text { inferior }(\mathrm{mm})\end{array}$ & $(18-) 22-35(-40)$ & $40-47(-52)$ \\
Lema* & Superficie & Glabra, o con nervios escábridos & Glabra \\
& Ápice & Con dos apéndices de 1-3 mm & Con dos apéndices de 3-4 mm \\
& Longitud(mm) & $(7-) 9-12(-14)$ & $14-15,5(-16,5)$ \\
Palea & Ápice & Ciliado & Pubescente y ciliado \\
& Longitud(mm) & $(6-) 7-10(-12)$ & $13-14,5(-15)$ \\
Arista & Longitud $(\mathrm{cm})$ & $(3,5-) 4,5-8,5(-10)$ & $7-10(-11)$ \\
& Columna & De 2/3 a completamente vilosa & De 1/2 a 2/3 vilosa
\end{tabular}

Tabla 1. Características morfológicas de las piezas florales en los taxones del género Macrochloa Kunth (Tribu Stipeae; Gramineae) que viven en Andalucía Oriental (España). ${ }^{*}$ La longitud de la lema no incluye el callo. Morphology of the flowers characters in the Macrochloa Kuth taxa (Tribu Stipeae; Gramineae) from Orient Andalusia (Spain). *The lemma dimension not includes the callus.

pelos de hasta 1,2 mm; pánicula 40-60(-70) $\mathrm{cm}$, densa; flores de hasta $8 \mathrm{~cm}$ con pedicelos de hasta $10 \mathrm{~mm}$, escábridos, rectos; glumas subiguales, agudas, glabras, la superior 32$40(-45) \mathrm{mm}$, la inferior 40-47(-52) $\mathrm{mm}$; callo $0,2-2,5(-3,5) \mathrm{mm}$, pubescente, punzante; lema $14-15,5(-16,5) \mathrm{mm}$, pubescente, bifida con dos prolongaciones membranosas en el ápice de 3-4 mm pubescentes; arista 7-10(11) $\mathrm{cm}$, unigeniculada, vilosa en la columna de $1 / 2$ a $2 / 3$ de su longitud, escábrida en el pico; palea 13-14,5(-15) mm, pubescente y ciliada en el ápice, binerviada, glabra en el dorso; anteras de hasta $16 \mathrm{~mm}$, pelosas en el ápice. Florece de Abril a Junio.

En la figura 1 (a y $a^{\prime}$ ) aparece representada una gluma y un antecio con arista.

Material estudiado: HS: ALMERÍA: Presa de Beninar, barranco de Río Grande, 2-5-1985, J. Guirado. ALME 17470 (Holotipo).
Clave para distinguir a las dos subespecie del Macrochloa tenacissima (Loefl. ex L.) Kunth, en Andalucía Oriental (España).

1. Lema de hasta $14 \mathrm{~mm}$. Columna de la arista cubierta de pelos largos de $2 / 3$ a totalmente. Panícula de 14-35(-45) $\mathrm{cm}$

M. tenacissima subsp. tenacissima

1. Lema de más de $14 \mathrm{~mm}$. Columna de la artista cubierta de pelos largos en $1 / 2$ a $2 / 3$ de su longitud. Panícula de más de $40 \mathrm{~cm}$

\section{M. tenacissima subsp. umbrosa}

\section{Stipa sección Stipa L.}

En la Península Ibérica existen tres especies endémicas de esta sección: Stipa apertifolia Martinovsky, Stipa iberica Martinovsky y Stipa pauneroana (Martinovsky) F.M. Vázquez \& Devesa, de las cuatro especies que conocemos para el territorio. En Andalucía Oriental, los seis taxones conocidos para la sección son endémicos de la Península. Como podemos 


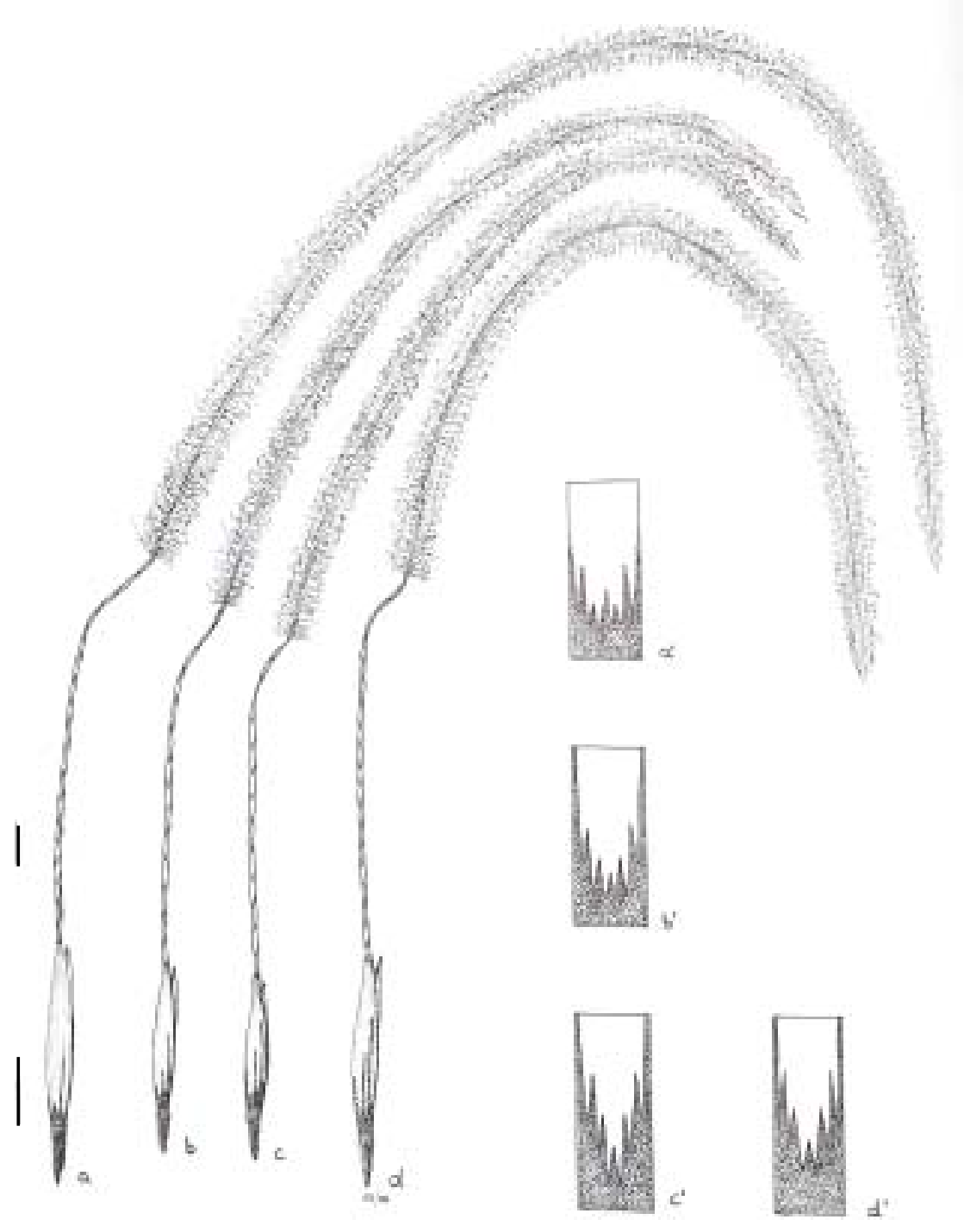

Figura 2. Antecio con arista y representación esquemática de la distribución de la melosidad en la lema de Stipa almerienses F.M. Vázquez sp. nov. (a, y'a'), Stipa apertifolia Martinovsky (b, y b'), Stipa irica Martinovsky (c, y c') y Stipa pauneroana (Martinovsky)F.M. Vázquez \& Devesa (d, y d'). Las barras indican $5 \mathrm{~mm}$. Anthecium with awn and schematic representation of the hairs lemma distribution for Stipa almerienses F.M. Vázquez sp. nov. (a, and a'), Stipa apertifolia Martinovsky (b, and b'), Stipa irica Martinovsky (c, and c') and Stipa pauneroana (Martinovsky)F.M. Vázquez \& Devesa (d, and d'). The bars are $5 \mathrm{~mm}$. 


\begin{tabular}{|c|c|c|c|c|c|}
\hline Características & & S. almeriensis & S. apertifolia & S. iberica & S. pauneroana \\
\hline \multirow[t]{3}{*}{ Limbo } & Adaxial & $\begin{array}{l}\text { Con pelos de más } 0,2 \\
\text { mm en los márgenes }\end{array}$ & $\begin{array}{l}\text { Escábrido, o con pelos de } \\
\text { más } 0,2 \text { toda superficie }\end{array}$ & $\begin{array}{l}\text { Escábrido, o con pelos de } \\
\text { más } 0,2 \text { toda superficie }\end{array}$ & $\begin{array}{l}\text { Con pelos de más } 0,2 \mathrm{~mm} \\
\text { en toda superficie }\end{array}$ \\
\hline & Abaxial & $\begin{array}{l}\text { Glabra o finamente } \\
\text { escábrida }\end{array}$ & $\begin{array}{l}\text { Glabra o esparcidamente } \\
\text { escábrida }\end{array}$ & $\begin{array}{l}\text { Fuertemente escábrida o } \\
\text { pubescente }\end{array}$ & Fuertemente escábrida \\
\hline & Diámetro(mm) & $(0,6-) 0,7-0,9(-1)$ & $(0,6-) 0,7-0,9(-1,1)$ & $0,3-0,5(-0,7)$ & $0,3-0,6(-0,7)$ \\
\hline \multirow[t]{2}{*}{ Lema* } & Líneas de pelos & $1 / 2$ a $2 / 3$ de su longitud & Toda su longitud & Toda su longitud & Toda su longitud \\
\hline & Longitud(mm) & $17-19(-21)$ & (13-) $14-15(-16)$ & $(12-) 13-15(-16)$ & $(16,5-) 17-19(-22)$ \\
\hline \multirow[t]{2}{*}{ Palea } & Ápice & Agudo & Agudo & A gudo o ciliado & Agudo o ciliado \\
\hline & Longitud(mm) & $14-17(-20)$ & $10-14(-15)$ & $11-14(-15)$ & $14-17(-19)$ \\
\hline Arista & Longitud (cm) & $(26-) 29-41(-46)$ & $(19-) 21-28(-32)$ & $(19-) 21-30(-35)$ & $(24-) 28-40(-45)$ \\
\hline
\end{tabular}

Tabla 2. Características morfológicas del limbo y las piezas florales en el grupo Stipa sección Stipa L., en Andalucía Oriental (España). * La longitud de la lema no incluye el callo. Morphology characteristics in the limb and flowers pieces for the Stipa section Stipa taxa from Orient Andalusia (Spain). *The lemma dimension not includes the callus.

entender la Sección Stipa del mismo género se encuentra representada en la Península Ibérica principalmente por taxones endémicos y en el SE del territorio se concentran buena parte de la diversidad de este grupo (Vázquez \& Devesa, 1997). El estudio pormenorizado del material de los herbarios previamente indicados, ha permitido encontrar un nuevo taxon para la sección Stipa en Andalucía Oriental, que posiblemente sea endémico de esa área. El nuevo taxon se le ha asignado la categoría de especie, y se diferencia con claridad del resto de especies de la sección por los caracteres florales y foliares, que aparecen reflejados en la tabla 2.

Stipa almeriensis F. M. Vázquez sp. nov. Holotipo: ALME 17433. HS: ALMERÍA: Minas de Almagrera, 1650 msm,19-06-1984, J. Guirado. Ejemplar derecho.

Diagnosis: Differt a Stipa apertifolia arista (26-)29-41(-46) (versus [19-]21-28[32]) $\mathrm{cm}$, et lemma $2 / 3$ usque $1 / 2$ pilosa (versus plene pilosam) et 17-19(-21) (versus [13-]14-15[-16]) mm longa.

Descripción: Plantas cespitosas de. 30-
$80 \mathrm{~cm}$; vaina de la hoja glabra a finamente escábrida; lámina $5-50 \mathrm{~cm}$, convoluta, pubescente en los márgenes del haz y glabra a finamente escábrida en el envés; lígula (4)5-12 mm, escábrida; pánicula 10-29 cm, laxa; flores con pedicelos de más de $10 \mathrm{~mm}$, pubescentes; glumas subiguales, setaceas, glabras, la superior (65-)70-90(-94) mm, la inferior (67-)75-95(-97) $\mathrm{mm}$; callo 5-7 mm, pubescente, punzante; lema 17-19,5(-21) $\mathrm{mm}$, con 7 líneas de pelos; arista (26-)2941(-46) cm, bigeniculada, columna glabra y pico plumoso con pelos de hasta $8 \mathrm{~mm}$; palea 14-17(-20) mm, aguda, glabra en el dorso; anteras de hasta $15 \mathrm{~mm}$, glabras; cariópside de hasta $16 \mathrm{~mm}$, fusiforme. Florece de Mayo a Julio.

En las figuras 2 y 3 aparecen representados el antecio con la arista y una sección del limbo de las hojas.

Material estudiado: HS: ALMERÍA: Minas de Almagrera, 1650 msm,19-06-1984, J. Guirado. ALME 17433 (Holotipo). Sierra de Gador, matorrales orófilos sobre suelos rocosos calcáreos, 1600 msm, 19-06-1992, A. Hervás. GDAC 37857. GRANADA: 

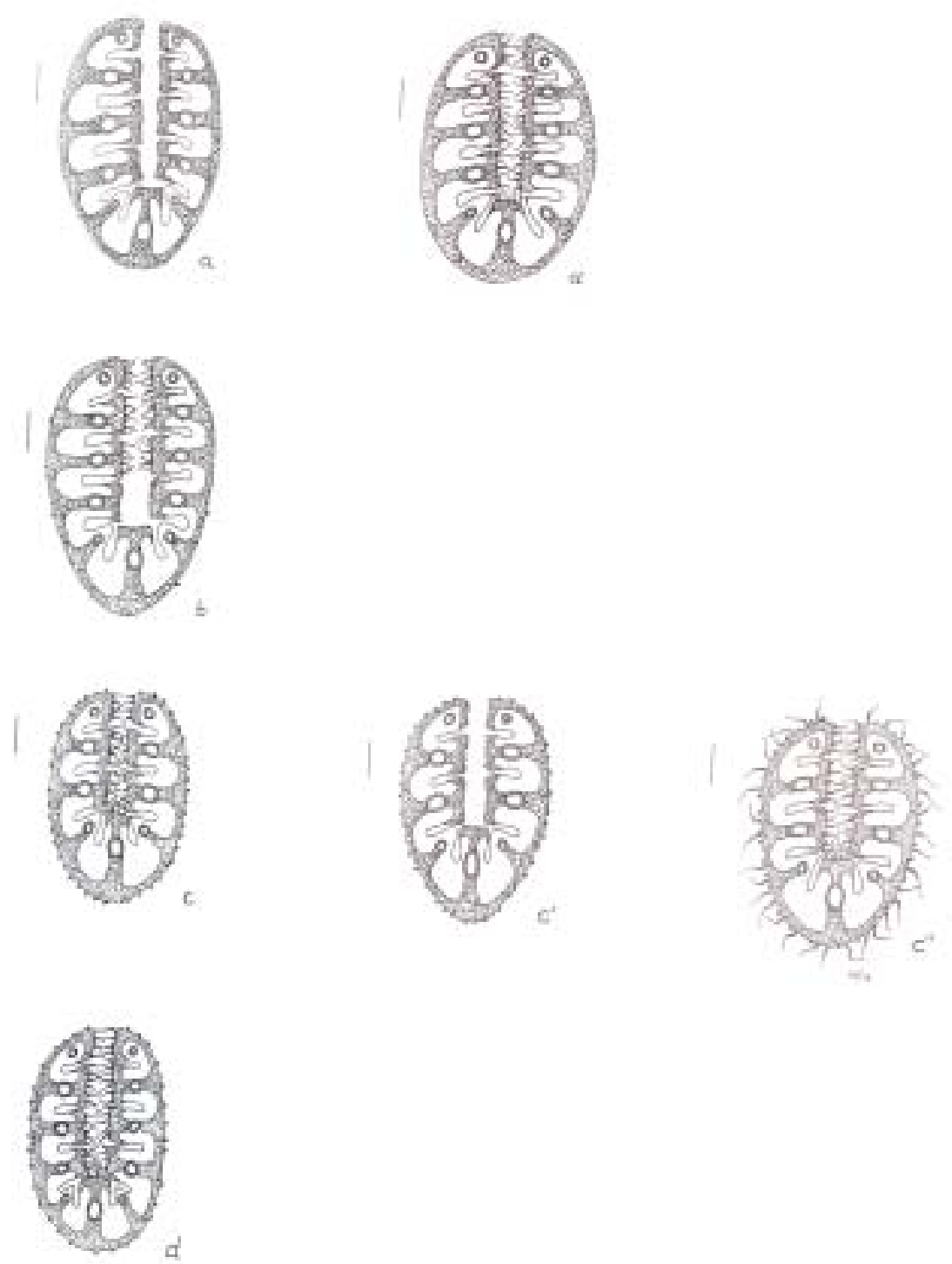

Figura 3. Sección de las hojas estériles para los taxones de la sección Stipa del género Stipa L., en Andalucía Oriental (España). a: Stipa apertifolia var. apertifolia Martinovsky; a': Stipa apertifolia var. nevadensis F.M. Vázquez \& Devesa; b: Stipa almeriensis F.M. Vázquez sp. nov.;'c: Stipa iberica var. iberica Martinosvky; c': Stipa iberica var. austro-iberica (H.Scholz)F.M. Vázquez \& Devesa; c": Stipa iberica var. pseudodasyphylla Martinovsky; y d: Stipa pauneroana (Martinovsky)F.M. Vázquez \& Devesa. Las barras indican 0,12 mm. Sterile leaves cross section in the Stipa section Stipa from Orient Andalusia (Spain): a: Stipa apertifolia var. apertifolia Martinovsky; a': Stipa apertifolia var. nevadensis F.M. Vázquez \& Devesa; b: Stipa almeriensis F.M. Vázquez sp. nov.; c': Stipa iberica var. iberica Martinosvky; c': Stipa iberica var. austro-iberica (H.Scholz) F.M. Vázquez \& Devesa; c": Stipa iberica var. pseudodasyphylla Martinovsky; and d: Stipa pauneroana (Martinovsky) F.M. Vázquez \& Devesa. The bars are 0,12 $\mathrm{mm}$. 
Sierra de Cazulas, próximo a la Casa Forestal, en prados, 07-06-1983, J. Guirado. GDAC 15576. Sierra de Baza, matorral heliófito degradación, 17-07-1984, sin colector, GDAC 26148.

Clave para distinguir a las especie de Stipa Sección Stipa L. en Andalucía Oriental (España).

1. Limbo de (0,6-)0,7-1,1 mm de diámetro, glabro o finamente escábrido en el envés

1. Limbo de $0,3-0,5(-0,7) \mathrm{mm}$ de diámetro... fuertemente escábrido o pubescente en el envés 3

2. Lema 17-19(-21) mm, con 7 líneas de pelos que no alcanzan el ápice. Arista (26-)29-41(46) $\mathrm{cm}$. Limbo en el haz pubescente

St. almeriensis

2. Lema (12-)13-15(-16) mm, con 7 líneas de pelos que algunas alcanzan el ápice. Arista de (19-)21-28(-32) cm. Limbo en el haz escábrido o pubescente St. apertifolia

3. Lema (16,5-)17-19(-22) mm Arista (24-)28$40(-45) \mathrm{cm}$ St. pauneroana 3. Lema (12-)13-15(-16) mm. Arista (19-)21-30($35) \mathrm{cm}$ St. iberica

AGRADECIMIENTOS. Deseo agradecer Al Dr. Ricardo Cabezas de Herrera su ayuda en la corrección de las diagnosis latinas.

\section{BIBLIOGRAFÍA}

BOLÓS, O. DE \& J. VIGO -1984/2001- Flora dels Països Catalans (4 vols.) Barcelona.

CASTROVIEJO, S. et al. -1986/2005- (eds.) Flora Ibérica (vols. 1-8, 14, 21). Madrid.

DEVESA, J.A. -1992- (ed.) Anatomía Foliar y palinológica de las gramíneas extremeñas. Badajoz.

DEVESA J.A. -1995- (ed.) Vegetación y Flora de Extremadura. Badajoz.

VALDÉS B., S. TALAVERA y E. GALIANO 1987- (eds.) Flora vascular de Andalucía Occidental (3 vols). Barcelona.
VÁZQUEZ F. M. y J. A. DEVESA -1997Revisión del género Stipa L. y Nassella Desv. (Poaceae) en la Península Ibérica e Islas Baleares. Acta Bot. Malacitana 21:125-189.

VÁZQUEZ F. M. \& M. E. BARKWORTH -2004Resurrection and emendation of Macrochloa (Gramineae: Stipeae). Bot. J. Linnean Society 144: 483-495.

VÁZQUEZ F.M., H. SCHOLZ y M. SONNENTAG -1999- Dos nuevas especies y una combinación en el género Stipa L., sección Leiostipa Dumort. (Poaceae) para el SE de España. Acta Bot. Malacinata 24: 2732.

VILLAR, L., J.A. SESÉ y J. V. FERNÁNDEZ 1997/2001-. Atlas de la Flora del Pirineo Aragonés (2 vols.). Angües (Huesca).

Aceptado para su publicación en octubre de 2006

Dirección del autor. Dpto. Producción Forestal, Centro de Investigación y Desarrollo Tecnológico, Finca La Orden, Apartado 22, 06080 Badajoz (España).E-mail: frvazquez50@hotmail.com 